\title{
Relationship between plasma apolipoprotein B concentrations and LDL particle number
}

This article was published in the following Dove Press journal:

Research Reports in Clinical Cardiology

18 September 2014

Number of times this article has been viewed

\author{
Pamela B Morris' \\ Kellie H McLain' \\ Hector A Malave ${ }^{2}$ \\ James A Underberg ${ }^{3}$ \\ Ngoc-Anh Le ${ }^{4}$ \\ Michael D Shapiro ${ }^{5}$ \\ Deborah A Winegar 6 \\ Ray Pourfarzib 6 \\ 'Division of Cardiology, Medical \\ University of South Carolina, \\ Charleston, SC, USA; ${ }^{2}$ Cardiology \\ of Atlanta, Atlanta, GA, USA; \\ ${ }^{3}$ Department of Medicine, New York \\ University Langone Medical Center, \\ New York, NY, USA; ${ }^{4}$ Atlanta Veterans \\ Affairs Medical Center, Decatur, GA, \\ USA; ${ }^{5}$ Oregon Health and Science \\ University, Portland, OR, USA; \\ ${ }^{6}$ LipoScience, Inc., Raleigh, NC, USA
}

Correspondence: Ray Pourfarzib LipoScience, Inc., 2436 Burton Road, 2500 Sumner Blvd,

Raleigh, NC 27616, USA

Tel +l 6783626247

Fax +I 4233328284

Email rpourfarzib@liposcience.com
Abstract: Many subjects with relatively normal, or even optimal, levels of low-density lipoprotein cholesterol (LDL-C) have increased atherogenic lipoprotein particle concentrations (apolipoprotein $\mathrm{B}$ [apoB] and LDL particle number [LDL-P] determined by nuclear magnetic resonance spectroscopy [NMR]). Numerous analyses have demonstrated that apoB and LDL-P predict the risk of future cardiovascular events more robustly than LDL-C, as estimated using the Friedewald equation. Little is known about the relationship between an individual's apoB and LDL-P level, and whether the relationship is comparable at different levels of LDL-C. The aim of this study was to examine the correlation between plasma apoB and LDL-P levels and specifically to evaluate the heterogeneity of LDL-P at low levels of apoB ( $<$ the 20 th population percentile $[78 \mathrm{mg} / \mathrm{dL}]$ ). Data were derived from a group of consecutive patients added to a large, single laboratory database (LipoScience, Inc.) during a 1-week period in which a standard lipid profile, apoB, and LDL-P levels were available. When risk categories were assigned to the subjects using the Framingham Offspring Study's population percentiles for apoB and LDL-P, there was good agreement between the two measures when LDL-C levels were high ( $\geq 160 \mathrm{mg} / \mathrm{dL}$ ). However, among individuals with low LDL-C and apoB, NMR analysis could identify a subgroup of individuals with potentially greater cardiovascular risk, as suggested by unexpected elevations in LDL-P.

Keywords: apolipoprotein B, LDL particle number, nuclear magnetic resonance spectroscopy, lipoproteins

\section{Introduction}

Low-density lipoprotein cholesterol (LDL-C) has been used as a predictor of cardiovascular risk and a marker of the efficacy of therapy for several decades. Recent evidence has demonstrated that LDL-C frequently underestimates risk among patients with metabolic disorders. ${ }^{1}$ Alternate measures of atherogenic apoB-containing lipoprotein particles, including LDL particle number (LDL-P) determined by nuclear magnetic resonance (NMR) spectroscopy or apolipoprotein B (apoB) levels, have been shown to provide a more reliable assessment of cardiovascular disease (CVD) risk, particularly in patients with metabolic dyslipidemic disorders who have achieved optimal LDL-C levels. ${ }^{2-4}$ Based on these findings, numerous expert consensus documents and international guidelines have endorsed the use of apoB and LDL-P in select populations..$^{3,4}$ While both apoB and LDL-P are highly correlated with each other, these two markers may be affected differently by various confounding factors that influence their ability to predict CVD.

In this report, we examined the relationship between plasma apoB and LDL-P levels in a cross-sectional study of 1,027 men and women; evaluating the heterogeneity of 
LDL-P at low and high levels of apoB $(<$ the 20th percentile $[78 \mathrm{mg} / \mathrm{dL}]$ and $>$ the 80 th percentile $[118 \mathrm{mg} / \mathrm{dL}]$ in the Framingham Offspring Study population).$^{5}$

\section{Methods}

Plasma samples were collected from fasting patients seen at physicians' offices during a 1-week period (December 29, 2010-January 5, 2011), according to sample preparation specifications required for NMR LipoProfile ${ }^{\circledR}$ (LipoScience Inc., Raleigh, NC, USA) analysis. Samples (one per patient for a total of 1,027) were shipped to LipoScience, Inc., and analyzed within 48 hours of collection. The information available for each patient was limited to sex and age; the patient's medical history and/or medication use was not available.

Plasma lipids (total cholesterol, triglycerides, and highdensity lipoprotein cholesterol [HDL-C]) were determined by enzymatic methods, using an Olympus AU680 ${ }^{\circledR}$ autoanalyzer, with reagent and calibrator kits supplied by Beckman Coulter Inc. (Brea, CA, USA). The LDL-C was calculated from total cholesterol and HDL-C using the Friedewald formula. ${ }^{6}$ The apoB concentrations were determined by an immunoturbidimetric method using reagents and standards from Beckman Coulter Inc. The lipoprotein particle concentrations were analyzed by NMR spectroscopy using a $400-\mathrm{MHz}$ proton NMR analyzer, as previously described. ${ }^{7}$

To allow comparisons between apoB and LDL-P, levels of each were expressed as population percentiles based on data from the Framingham Offspring Study., ${ }^{2,5}$ The Framingham Offspring Study was chosen because it is the only large observational study in which both apoB and LDL-P population percentiles were reported. Discordance was defined as a mismatch between apoB and LDL-P percentiles. For example, an individual whose apoB level is in the $<20$ th percentile and LDL-P level is in the 50th percentile would be considered discordant.

\section{Results}

Table 1 presents the clinical characteristics of the 533 men and 494 women included in this cross-sectional observational study. The mean LDL-C across the entire population was $112.7 \pm 39.4 \mathrm{mg} / \mathrm{dL}$, mean LDL-P was $1,590 \pm 554 \mathrm{nmol} / \mathrm{L}$, and mean apoB was $97.7 \pm 25.1 \mathrm{mg} / \mathrm{dL}$. Figure 1 illustrates the linear relationship between the concentration of apoB determined by immunoassay and LDL-P assessed by NMR spectroscopy for men (Figure 1A) and women (Figure 1B). As shown, the relationship between these two measurements was comparable, independent of sex.
Table I Subject characteristics, mean (standard deviation)

\begin{tabular}{|c|c|c|c|}
\hline Parameters & Women & Men & All \\
\hline Sample size & 494 & 533 & $\mathrm{I}, 027$ \\
\hline Age (years) & $57.2(14.2)$ & $60.0(14.6)$ & $58.5(\mid 4.5)$ \\
\hline Total chol (mg/dL) & $203.0(47.0)$ & I85.2(43.4) & $193.7(46.0)$ \\
\hline TG (mg/dL) & I $58.8(87.4)$ & I75.6 (I54.0) & $167.5(126.7)$ \\
\hline HDL-C (mg/dL) & $53.5(12.6)$ & $45.3(10.3)$ & $49.3(12.4)$ \\
\hline Non-HDL-C (mg/dL) & I $49.4(44.8)$ & I39.7 (42.5) & | 44.4 (43.9) \\
\hline Calc LDL-C (mg/dL) & II 7.7 (39.9) & $107.8(38.4)$ & I I 2.7 (39.4) \\
\hline ApoB (mg/dL) & $100.5(25.8)$ & $95.1(24.2)$ & $97.7(25.1)$ \\
\hline LDL-P (nmol/L) & $\mathrm{I}, 630(563)$ & I,554 (544) & I,590 (554) \\
\hline
\end{tabular}

Abbreviations: total chol, total cholesterol; TG, triglycerides; HDL-C, high-density lipoprotein cholesterol; non-HDL-C, nonhigh-density lipoprotein cholesterol; calc LDL-C, calculated low-density lipoprotein cholesterol; apoB, apolipoprotein B; LDL-P, low-density lipoprotein particle number.

Figure 2 shows the distribution of LDL-P values in subjects with an apoB concentration $<78 \mathrm{mg} / \mathrm{dL}$ which corresponds to the 20th Framingham Offspring Study population percentile $(\mathrm{n}=228)$. At these low apoB concentrations, only $64 \%$ had comparably low LDL-P concentrations of $<1,100 \mathrm{nmol} / \mathrm{L}$;
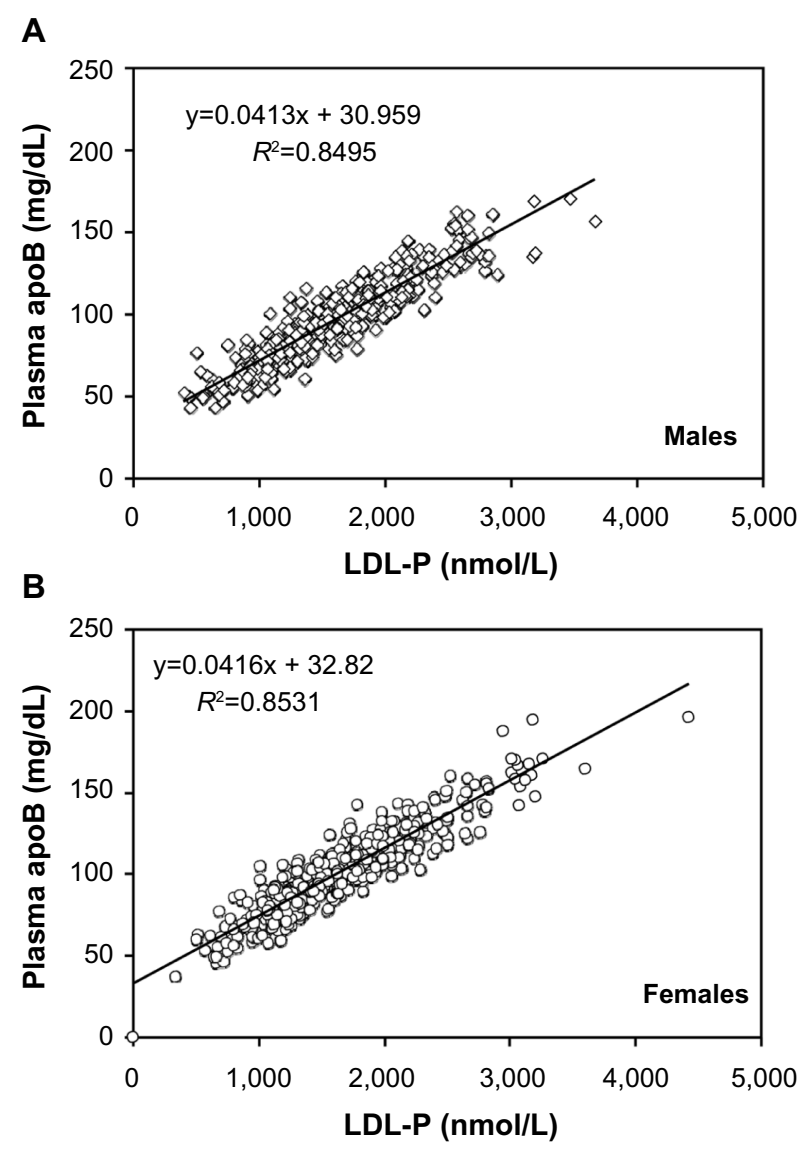

Figure I Relationship between apoB concentrations and LDL-P is independent of sex.

Notes: Relationship between plasma apoB determined by immunoassay and LDL-P determined by NMR spectroscopy. Linear regression between apoB and LDL-P for men (A) and women (B).

Abbreviations: apoB, apolipoprotein B; LDL, low-density lipoprotein; LDL-P, lowdensity lipoprotein particle number; NMR, nuclear magnetic resonance. 


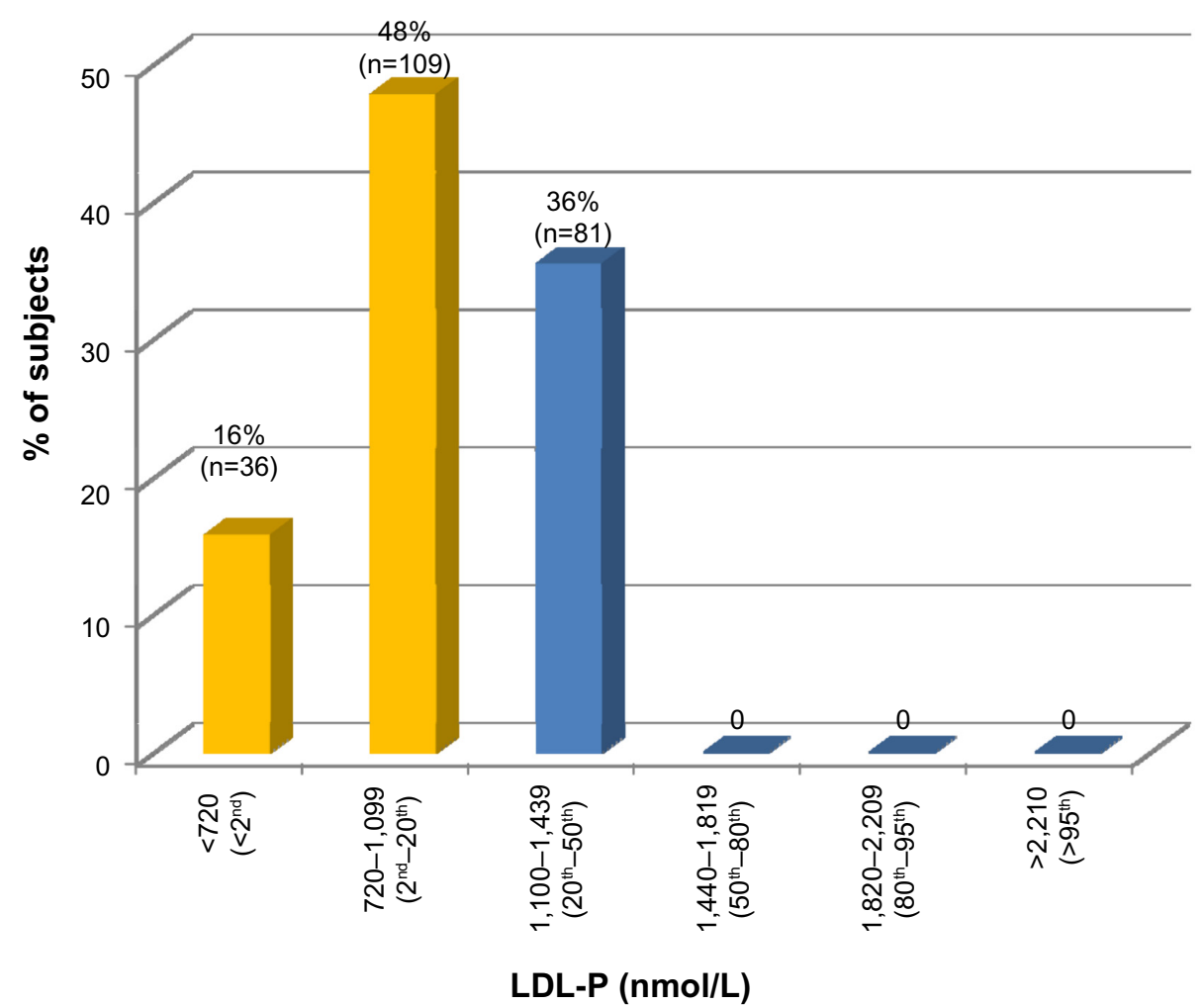

Figure 2 LDL-P distribution in subjects with apoB levels $<78 \mathrm{mg} / \mathrm{dL}$.

Note: Numbers in brackets refer to the percentile ranks within the Framingham Offspring Study population.

Abbreviations: LDL-P, low-density lipoprotein particle number; apoB, apolipoprotein B.

$36 \%$ had LDL-P levels of 1,100-1,439 nmol/L, which corresponds to the 20th-50th Framingham Offspring Study population percentile. In contrast, in subjects with apoB concentrations $>118 \mathrm{mg} / \mathrm{dL}$ (which corresponds to the 80 th Framingham Offspring Study population percentile [n=230]), $93 \%$ had comparably high LDL-P values $(\geq 1,820 \mathrm{nmol} / \mathrm{L})$ (Figure 3).

Figure 4 compares the distribution of various measures of LDL (LDL-C, LDL-P, and apoB) across Framingham Offspring Study population percentiles. While $40 \%$ of the subjects had LDL-C levels $<100 \mathrm{mg} / \mathrm{dL}$ (20th Framingham Offspring Study population percentile), only $17 \%$ and $22 \%$ had comparably low LDL-P and apoB levels $(<1,100 \mathrm{nmol} / \mathrm{L}$ and $<78 \mathrm{nmol} / \mathrm{L}$, respectively). Figure 5 shows the distribution of LDL-P levels among a subset of patients in the database (subjects) that had LDL-C levels $<100 \mathrm{mg} / \mathrm{dL}, 61 \%$ were found to have discordantly high LDL-P levels; whereas, $46 \%$ had discordantly high apoB levels.

\section{Discussion}

Recent studies suggest that $\mathrm{apoB}^{8}$ may be a better predictor of CVD risk than total cholesterol, LDL-C, ${ }^{9,10}$ and nonHDL-C. ${ }^{11,12}$ ApoB is the primary protein component of LDL particles, but it is also found in plasma in association with triglyceride-rich very low density lipoproteins, intermediate-density lipoproteins (IDL) and lipoprotein(a). The concentration of apoB in plasma is most commonly determined in commercial clinical laboratories by immunoturbidimetric and immunonephelometric methods that provide a single measure for both the liver-derived and intestinally derived forms of apoB (apoB100 and apoB48, respectively). In nonfasting samples, the measured apoB may also include protein associated with chylomicrons and their remnants. It is not clear whether all apoB-containing lipoprotein particles have the same atherogenic potential as LDL particles.

Furthermore, this heterogeneity in apoB-containing particles may potentially interfere with the immunological recognition of the antigenic sites used for apoB quantitation. It is possible that variations in the folding of the apoB molecule between small and large LDL particles and differences in the concentrations of other lipids, such as phospholipids on the particle surface, may interfere with detection by immunoassay.

NMR spectroscopy offers an alternate method for the direct determination of the number of LDL particles in plasma. When placed in a magnetic field, the terminal methyl groups on lipoprotein-associated lipids emit a characteristic 


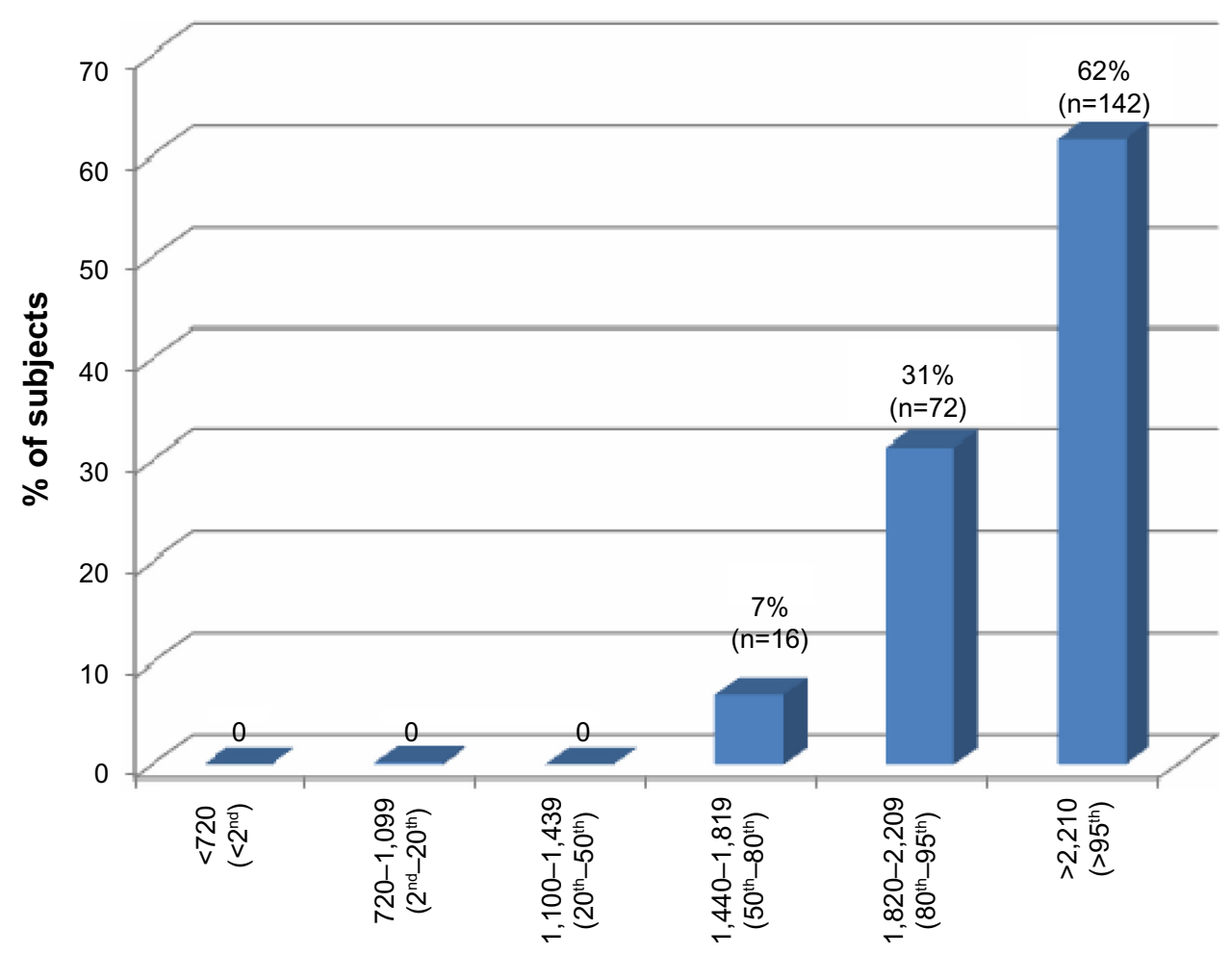

\section{LDL-P (nmol/L)}

Figure 3 LDL-P distribution in subjects with apoB levels $>118 \mathrm{mg} / \mathrm{dL}$.

Note: $A$ poB levels $>118 \mathrm{mg} / \mathrm{dL}$ correspond to the $=80$ th percentile for the Framingham Offspring Study population.

Abbreviations: LDL-P, low-density lipoprotein particle number; apoB, apolipoprotein B.

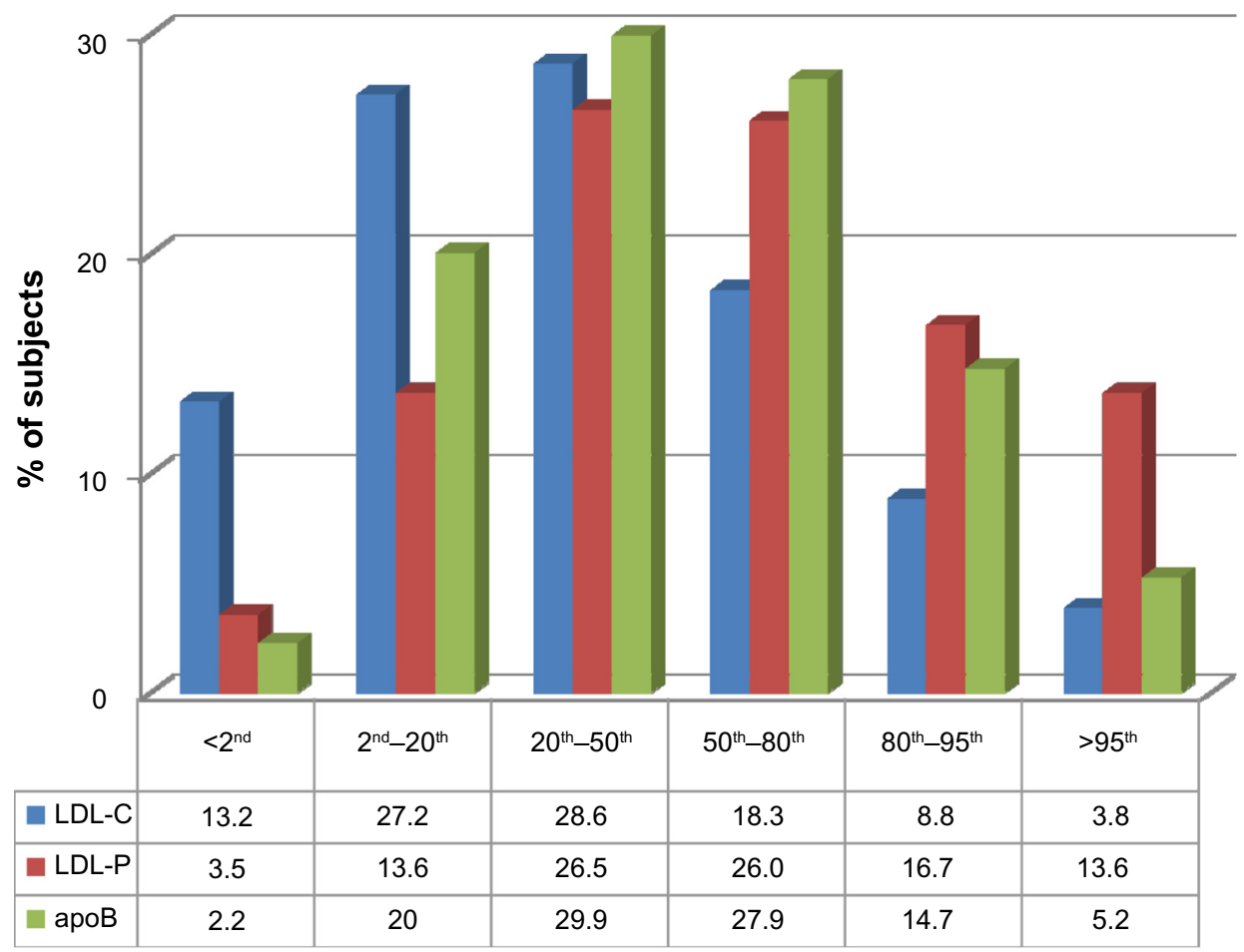

Figure 4 Distribution of LDL-C, LDL-P, and apoB levels across subjects based on population percentiles for the Framingham Offspring Study population. Abbreviations: LDL-C, low-density lipoprotein cholesterol; LDL-P, low-density lipoprotein particle number; apoB, apolipoprotein B. 


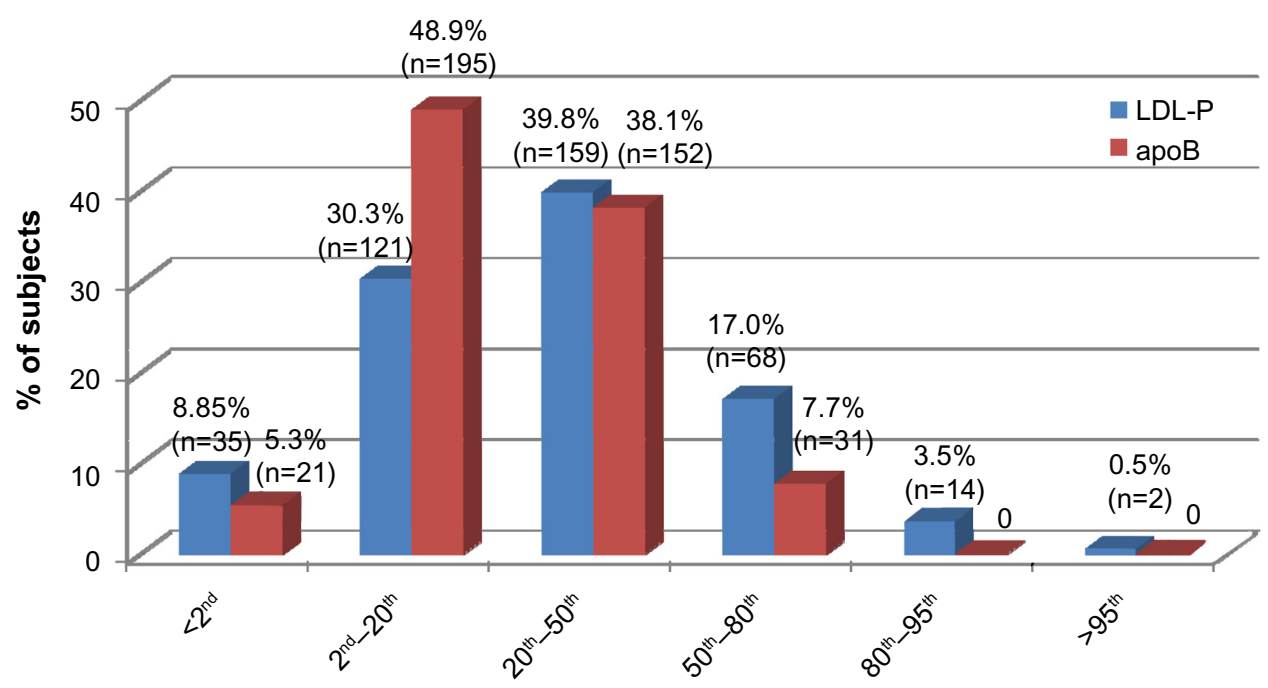

Framingham population percentile for LDL-P or apoB

Figure 5 Distribution of LDL-P and apoB levels in subjects with LDL-C levels $<100 \mathrm{mg} / \mathrm{dL}$ based on population percentiles for Framingham Offspring Study population. Abbreviations: LDL-C, low-density lipoprotein cholesterol; LDL-P, low-density lipoprotein particle number; apoB, apolipoprotein B.

NMR signal which varies depending on the size of the lipoprotein particle. ${ }^{7}$ The amplitude of the signal is directly proportional to the number of particles. ${ }^{7}$ Total LDL-P is determined by summing the concentrations of the individual LDL subclasses, including IDL. Numerous studies have reported that LDL-P is superior to LDL-C for the identification of patients at CVD risk as well as adjudicating the efficacy of lipid-modifying therapy. ${ }^{1}$ The American Association of Clinical Chemistry Lipoprotein and Vascular Diseases Division Working Group on Best Practices recently published a review of 25 clinical studies in which the strength of association of apoB and LDL-P with specific cardiovascular outcomes was evaluated. ${ }^{3}$ LDL-P measured by NMR was more strongly and more frequently associated with the clinical outcomes than apoB. ${ }^{3}$ The working group noted several advantages of LDL-P by NMR over apoB. NMR can provide information on other major lipoprotein classes, such as very low density lipoproteins, IDL, and HDL - in addition to LDL-P.

Furthermore, the NMR measurement of LDL-P appears to be more precise than that of apoB. The typical intra-assay coefficient of variation for apoB ranges from $5 \%-11 \%$; whereas, LDL-P has a coefficient of variation that ranges from $2 \%-4 \%{ }^{3}$

In the recently published Guideline on the Treatment of Blood Cholesterol to Reduce Atherosclerotic Risk in Adults, issued by the American College of Cardiology and the American Heart Association, ${ }^{13}$ LDL lowering remains a central tenet of clinical practice. The measurement of LDL continues to be relevant to guide the clinical judgment of patient adherence to treatment, individual response to therapy, and the need for adjustment in medications to achieve an appropriate individual response. This requires highly reliable LDL measures that correlate with clinical outcomes.

In the current report, we evaluated the relationship between apoB and LDL-P and their associations with conventional lipid biomarkers in a group of patients being followed at typical medical offices. Although many subjects had concordant levels of LDL-P and apoB, a considerable percentage with low apoB levels $(<78 \mathrm{mg} / \mathrm{dL})$ had higher than desirable LDL-P levels. These patients may retain considerable potential LDL-attributable CVD risk, with $36 \%$ of them having an LDL-P concentration $>1,100 \mathrm{nmol} / \mathrm{L}$. This discrepancy between LDL-P and apoB was not observed in subjects with high apoB levels $(>118 \mathrm{mg} / \mathrm{dL})$, as a similar percentage had comparably high LDL-P levels.

Further evidence that the apoB and LDL-P levels may provide different information related to the CVD risk was confirmed when the population percentiles for each of the measures from the Framingham Offspring Study were used to classify risk. Among the subjects with safe LDL-C levels $(<100 \mathrm{mg} / \mathrm{dL}$; the 20th Framingham Offspring Study percentile cut-point), $46 \%$ had discordantly high apoB levels; whereas, $61 \%$ had discordantly high LDL-P levels. This finding suggests that both markers uncovered excessive atherogenic particles in subjects who attained an 
LDL-C $<100 \mathrm{mg} / \mathrm{dL}$ and that management of this risk with more aggressive lipid-modifying therapy may be considered. This study additionally highlights the fact that apoB and LDL-P are not comparable measures of atherogenic lipoproteins, particularly at low levels of apoB, and suggests that one measure cannot simply be substituted for the other. LDL-P appears to identify significantly more individuals with excessive concentrations of atherogenic lipoproteins at low levels of both LDL-C and apoB and thus may have the potential to identify more subjects with residual cardiovascular risk. The hypothesis that LDL-P is a more robust predictor of atherogenic lipoprotein-related risk than apoB merits further investigation.

A limitation of this study is the lack of clinical information from study subjects, including medication utilization and associated comorbidities. Additionally, while this study demonstrates discordance between these two parameters at low levels of apoB, this investigation did not assess the superiority of one measure over the other at predicting CVD risk. Despite these limitations, this study clearly establishes that apoB and LDL-P are not comparable measures of atherogenic lipoproteins, particularly in individuals who might otherwise be considered low-risk having achieved optimal LDL-C levels.

\section{Disclosure}

Pamela B Morris is a member of the speaker's bureau for Abbott Laboratories, LipoScience, Inc., and Merck \& Co. Hector A Malave is a member of the speaker's bureau for LipoScience, Inc., and Abbott Laboratories. James A Underberg is: a consultant for Aegerion Pharmaceuticals, Amarin Corporation, Genzyme, LipoScience Inc., and Sanofi; a member of the speaker's bureau for Abbott Laboratories, Amarin Corporation, AstraZeneca, Daiichi Sankyo Inc., Eli Lilly and Company, Forest Pharmaceuticals Inc., GlaxoSmithKline, Kowa Pharmaceuticals, and Merck \& Co; and the recipient of research grants from Kowa Pharmaceuticals and LipoScience, Inc. Ngoc-Anh Le received a research grant from Merck \& Co and is a consultant for LipoScience, Inc. Michael D Shapiro is a member of the speaker's bureau for
LipoScience, Inc. Deborah A Winegar and Ray Pourfarzib are both full-time employees of LipoScience, Inc. Kellie $\mathrm{H}$ McLain reports no conflicts of interest in this work.

\section{References}

1. Rosenson RS, Davidson MH, Pourfarzib R. Underappreciated opportunities for low-density lipoprotein management in patients with cardiometabolic residual risk. Atherosclerosis. 2010;213(1):1-7.

2. Contois JH, McConnell JP, Sethi AA, et al; AACC Lipoproteins and Vascular Diseases Division Working Group on Best Practices. Apolipoprotein B and cardiovascular risk: position statement from the AACC Lipoproteins and Vascular Disease Division Working Group on Best Practices. Clin Chem. 2009;55(3):407-419.

3. AACC Lipoproteins and Vascular Diseases Division Working Group on Best Practices; Cole TG, Contois JH, Csako G, et al. Association of apolipoprotein $\mathrm{B}$ and nuclear magnetic resonance spectroscopy-derived LDL particle number with outcomes in 25 clinical studies: assessment by the AACC Lipoprotein and Vascular Diseases Division Working Group on Best Practices. Clin Chem. 2012;59(5):752-770.

4. Davidson MH, Ballantyne CM, Jacobson TA, et al. Clinical utility of inflammatory markers and advanced lipoprotein testing: advice from an expert panel of lipid specialists. J Clin Lipidol. 2011;5(5):338-367.

5. Cromwell WC, Otvos JD, Keyes MJ, et al. LDL Particle Number and Risk of Future Cardiovascular Disease in the Framingham Offspring Study - Implications for LDL Management. J Clin Lipidol. 2007;1(6):583-592.

6. Friedewald WT, Levy RI, Fredrickson DS. Estimation of the concentration of low-density lipoprotein cholesterol in plasma, without the use of the preparative ultracentrifuge. Clin Chem. 1972;18(6):499-502.

7. Jeyarajah EJ, Cromwell WC, Otvos JD. Lipoprotein particle analysis by nuclear magnetic resonance spectroscopy. Clin Lab Med. 2006;26(4): 847-870.

8. Stein EA, Sniderman A, Laskarzewski P. Assessment of reaching goal in patients with combined hyperlipidemia: low-density lipoprotein cholesterol, non-high-density lipoprotein cholesterol, or apolipoprotein B. Am J Cardiol. 2005;96(9A):36K-43K.

9. Kastelein JJ, van der Steeg WA, Holme I, et al; TNT Study Group; IDEAL Study Group. Lipids, apolipoproteins, and their ratios in relation to cardiovascular events with statin treatment. Circulation. 2008;117(23):3002-3009.

10. Levinson SS. Comparison of apolipoprotein B and non-high-density lipoprotein cholesterol for identifying coronary artery disease risk based on receiver operating curve analysis. Am J Clin Pathol. 2007;127(3): 449-455.

11. Barter PJ, Ballantyne CM, Carmena R, et al. ApoB versus cholesterol in estimating cardiovascular risk and in guiding therapy: report of the thirty-person/ten-country panel. J Intern Med. 2006;259(3):247-258.

12. Grundy SM. Use of emerging lipoprotein risk factors in assessment of cardiovascular risk. JAMA. 2012;307(23):2540-2542.

13. Stone NJ, Robinson JG, Lichtenstein AH, et al. 2013 ACC/AHA Guideline on the Treatment of Blood Cholesterol to Reduce Atherosclerotic Cardiovascular Risk in Adults: A Report of the American College of Cardiology/American Heart Association Task Force on Practice Guidelines. J Am Coll Cardiol. 2014;63(25 Pt B): 2889-2934.
Research Reports in Clinical Cardiology

\section{Publish your work in this journal}

Research Reports in Clinical Cardiology is an international, peerreviewed, open access journal publishing original research, reports, editorials, reviews and commentaries on all areas of cardiology in the clinic and laboratory. The manuscript management system is completely online and includes a very quick and fair peer-review system.

\section{Dovepress}

Visit http://www.dovepress.com/testimonials.php to read real quotes from published authors. 